\title{
Realization of face image recognition system based on histogram Yurong Zhang ${ }^{1,2}$ \\ ${ }^{1}$ School of information engineering, Wuhan University of Technology, Hubei Wuhan 430070, China \\ ${ }^{2}$ Electronics information Department, HuiShang vocational Technical college, Hefei Anhui 230061, China.
}

Keywords: histogram; face recognition; image processing; MATLAB

\begin{abstract}
In this paper, the face image recognition in the application of MATLAB to preprocess the image, application the toolbox classic image processing of the image, through the example to apply MATLAB image processing function, of a given face image processing, and applied to the face recognition system. In this paper, in summing up the face recognition system of analysis of several commonly used image pre processing method based, using MATLAB to realize a set a variety of pre processing method in one of the generic face image pre processing simulation system, the system as the image pre processing module can be embedded in the face recognition system, and the use of image gray histogram Alignment to achieve the recognition of human face images.
\end{abstract}

\section{Introduction}

System for the automatic recognition of face images compared with the fingerprint identification system, DNA identification of more convenience, because of its convenience sampling, can not contact the target identified and practical significance to research and development of the greater. Face images which are affected by many factors interference, the diversity of facial expression and the external imaging process of light, image size, rotation, posture change. The same individuals in different environment shoot the face images of different, sometimes very different. To identify bring great difficulty. Therefore under various disturbance conditions to achieve the face image recognition. It is more challenging.

\section{Identification System}

\section{Face image acquisition}

Generally speaking, the image is obtained through the camera uptake, but the uptake of image can be real can also be is the picture of the face or to relatively simple, can not consider the image through the camera to intake of the head, but directly to the given to identify.

Face detection

Face detection task is to determine whether there is a face in the static image. If there is a face, given the coordinates of the location in the image, the size of the face region information. The face tracking is required further output the detected position of the face, the size of the state with continuous time change.

Feature extraction

Through facial feature points detection and calibration that can be used to determine the significant feature points in face images (such as the eyes, eyebrows, nose, and mouth organ) position, but also can get the description of the shape information of these organs and facial contour.

According to the results of facial feature points detection and calibration, the facial features are described by some operations (including global and local features, explicit features and statistical features).

\section{Identity recognition based on face image matching}

The problem of face recognition, face identification). The input facial image and 3D face 
database of all known prototype face image similarity calculation and the sort of identity information of the input face is given. This includes two class recognition problem: a class is a closed set (close set face recognition problem, which assumes that the input face is a individual in the face database; another kind is open sets (open set recognition. The first step is to the input face is in a known face database judge. If it is, given its identity.

The face verification (face verification problem. The system in the input face image and input a user declared the face identity information, the system should be the identity of the input face image and claimed that identity is in conformity with the judge.

\section{Image processing functions Matlab implementation examples}

\section{Image type conversion}

Because behind the image enhancement, edge detection is used for gray image, and our image is RGB image. So first of all we want to on the original image type conversion. To achieve the following code:

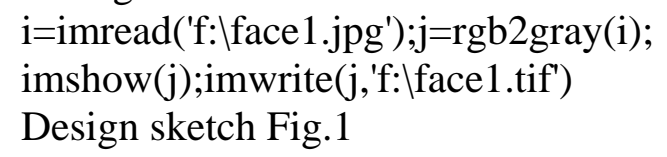

\section{Image enhancement}

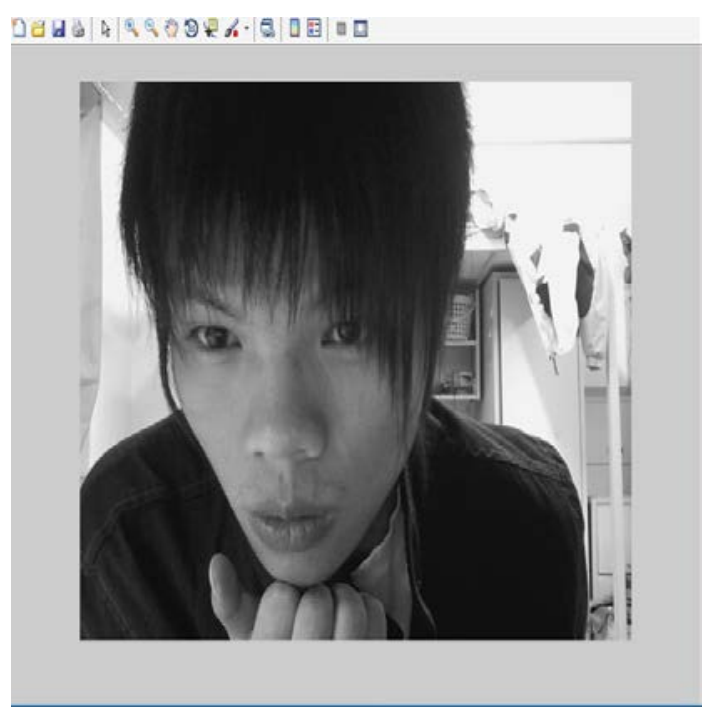

Fig.1 Design sketch

1) gray image histogram equalization

Through the comparison of the original image and the histogram equalization of visible image, the image becomes clearer, and after equalization histogram more desirable than the shape of the original histogram. This part of the code are as follows:

i=imread('f:Iface1.tif');

$\mathrm{j}=$ histeq(i);imshow(j);

figure,subplot(1,2,1),imhist(i);

subplot(1,2,2),imhist(j)

After the implementation of the effect diagram as shown in Figure 2 and figure 3.

2) gray image smoothing and sharpening

The purpose of smoothing filter is blurred image and eliminate noise, Matlab Image Processing Toolbox provides medfilter2 () function is used to realize the median filtering, wiener2 () to realize the adaptive filter to the noise image. In this example, in order to make the filtering effect is more obvious, we advance image think increase filtering, and adaptive filtering method for image filtering, sharpening is emphasize the details of the image is fuzzy, in this case using the predefined Gaussian filter method of image sharpening filter. Function of the realization of the code are as follows: 
i=imread('f:|fae1.tif');

j=imnoise(i,'guassian',0,0.02);

subplot(1,2,1),imshow(j);

j1=wiener2(j); subplot(1,2,2),imshow(j1);

$\mathrm{h}=$ fspecial('gaussian',2,0.05);j2=imfilter(i,h);figure,subplot(1,2,1),imshow(i)

subplot(1,2,2),imshow(j2)

The obtained results are shown in Figure 4 and 5.

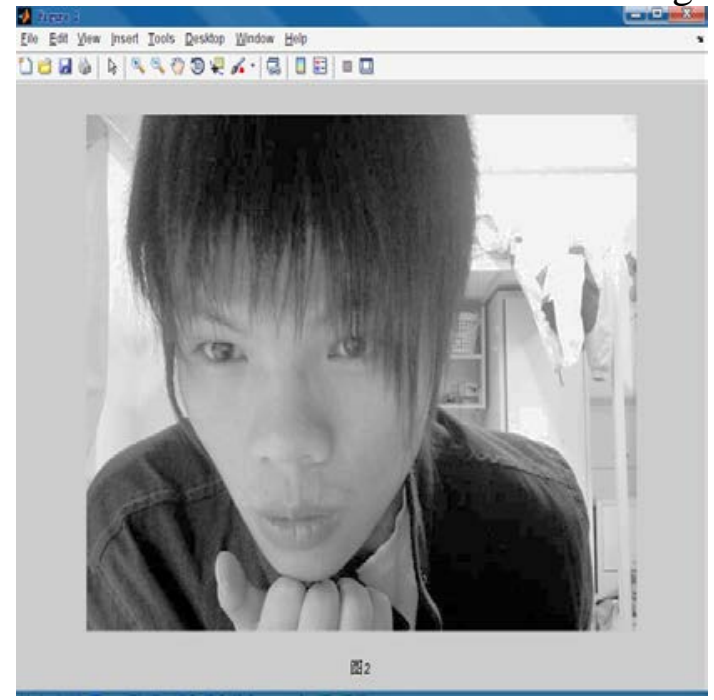

Fig. 2 gray image after equalization

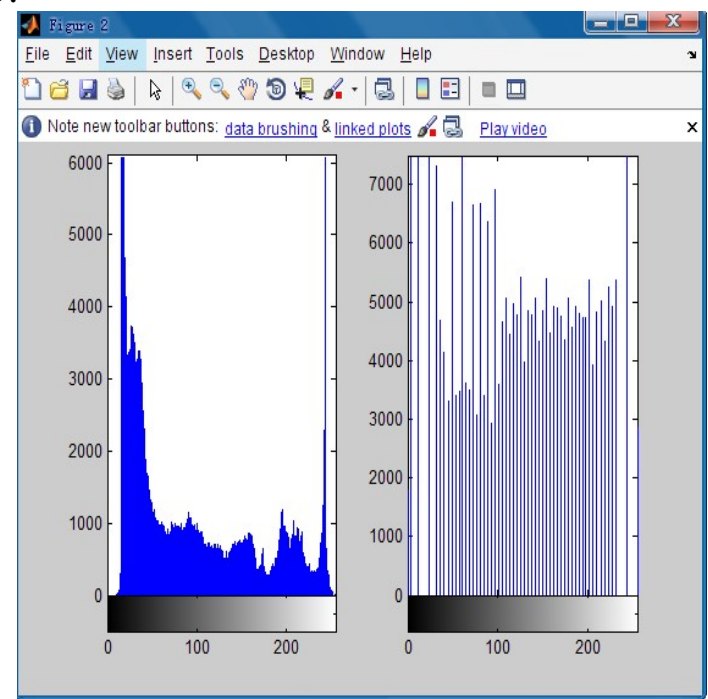

Fig. 3 histogram comparison chart before and after equalization
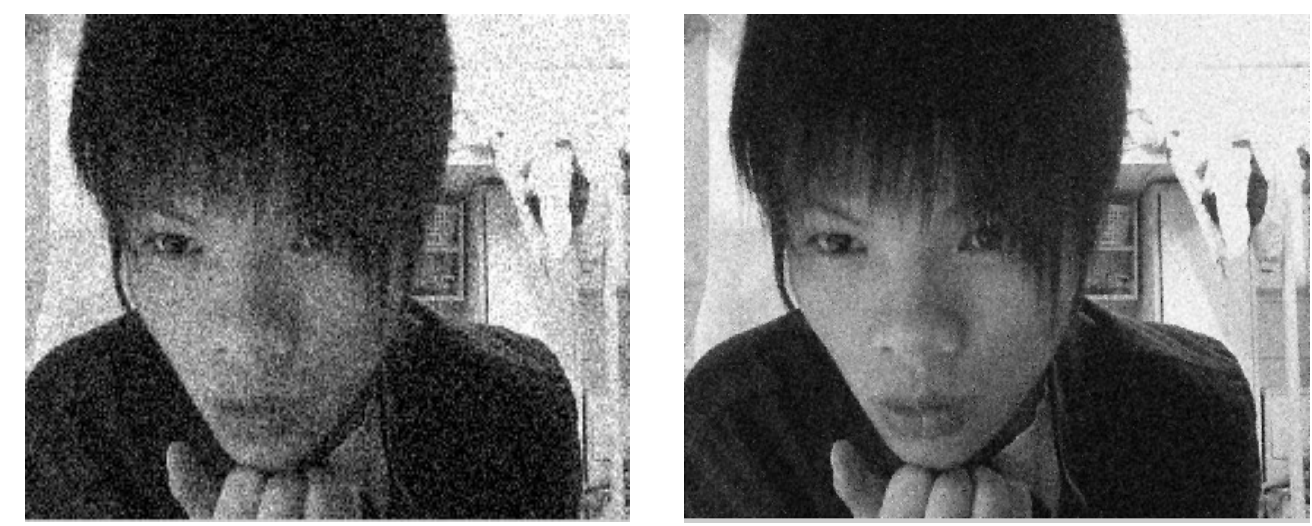

Fig.4 smoothing filtering effect
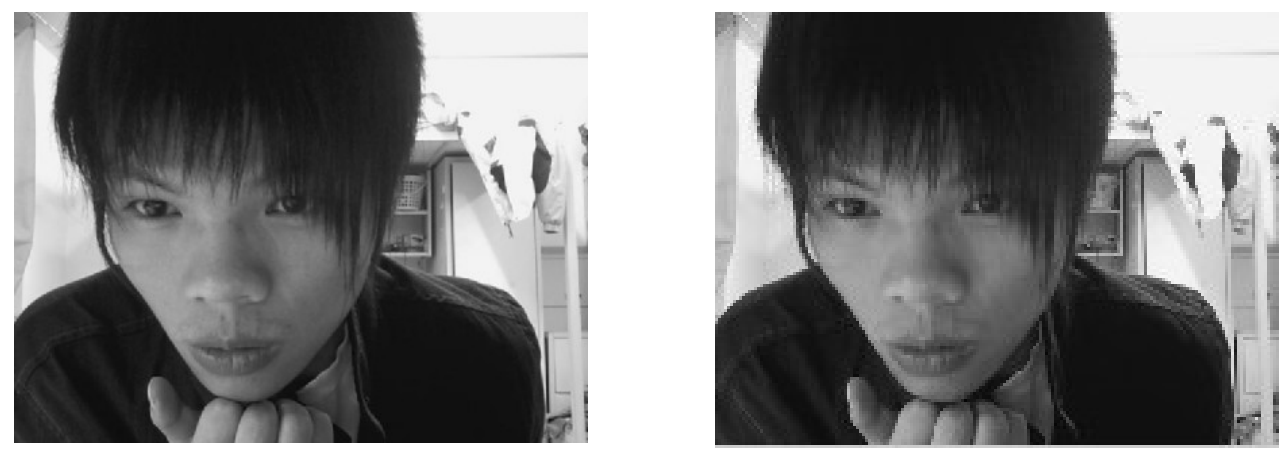

Fig.5 Effect of sharpening filter

3)Matlab7.0 Image Processing Toolbox provides edge () function to achieve edge detection, but also there are various methods for selecting operators, in this case, the Canny operator is used to carry out edge detection.

Program code is as follows:

i=imread('f:face.tif');

j=edge(i,'canny',[0.04,0.25],1.5); 
$\operatorname{imshow}(\mathrm{j})$

Effect diagram as shown in figure 6.

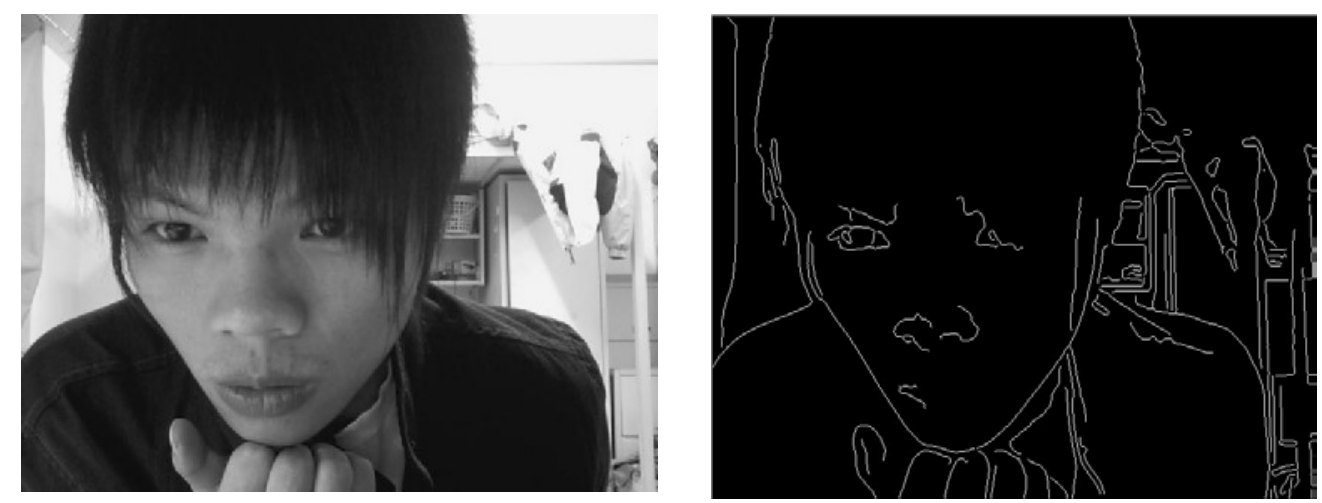

Fig.6 Effect of edge detection

\section{Face image preprocessing}

Meet the needs and sources of different face recognition system based on the image recognition algorithm, the preprocessing methods are also different. Commonly used face image preprocessing methods: filtering denoising, gray transform, image binary, edge detection, size normalization and gray one. In the same system may only have one kind of or several pretreatment methods, but once the library collection to the original image quality have great changes (such as face size, light intensity, shooting conditions, imaging system changes, the original pretreatment module will not feature extraction, but also update, which is very inconvenient. In view of this , author summarizes and analyses the filtering and denoising, edge detection, gray level transformation three widely for different face recognition system in the pre processing method, design a general face image pre processing system simulation. The system can be in different conditions of the original image, the corresponding pretreatment. For example, the user can choose according to need to use different filtering methods to remove noise, different edge detection operators face detection edge, choose different gray transform algorithms achieve the gray-scale image normalization and gray correction, simulation system but also the realization of the size of the normalized, binary and other common image pre processing algorithm.

\section{Implementation of face recognition based on Matlab}

Achieve results as shown in Figure 7 and 8.

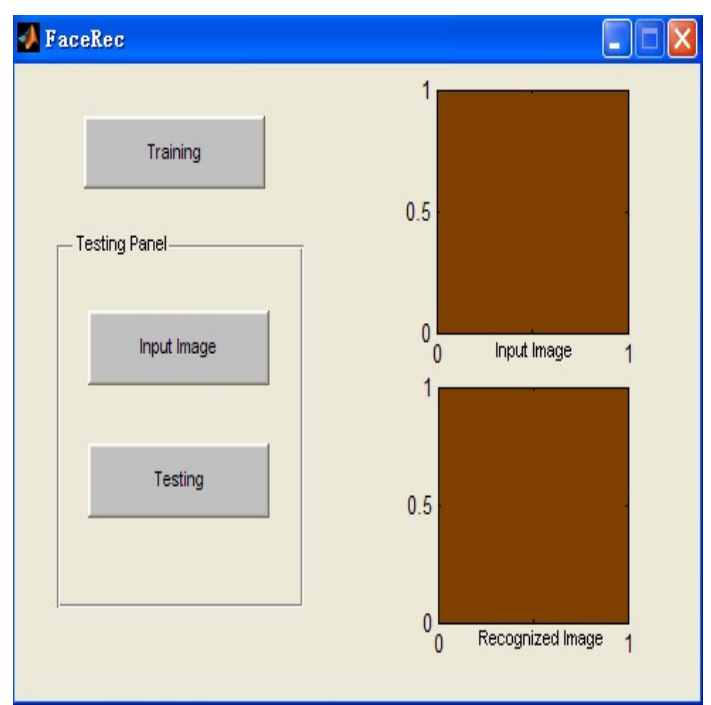

Fig.7 user interface

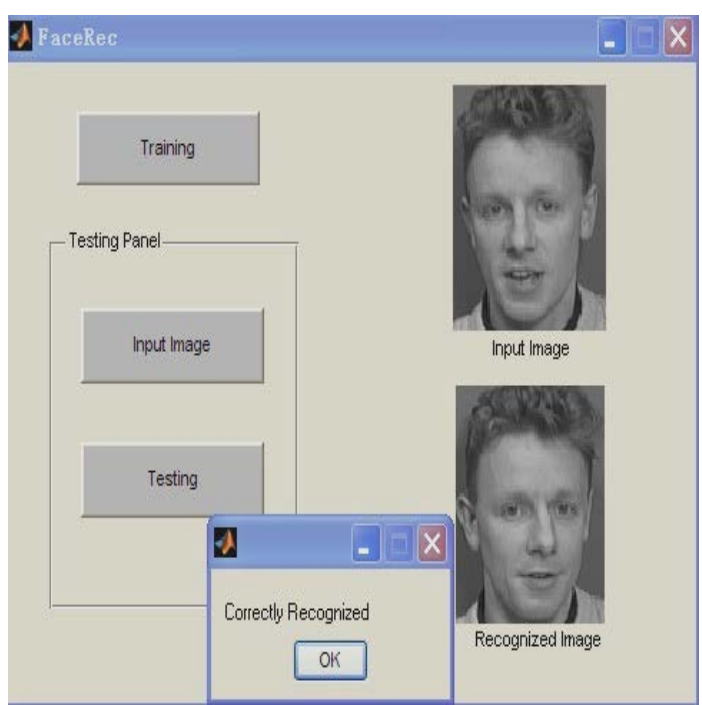

Fig.8 implementation results 


\section{Conclusion}

Matlab digital image processing and recognition system is actually a wide range of applications, the author is only targeted, there is a choice of some development and implementation.

The main function modules of the system are two: (1) the basic method of digital image processing; (2) face recognition.

In "digital image processing of the basic methods of this part, used some basic methods and some of the most commonly used processing method to achieve image pre processing, such as format conversion, the gray level changes and sharpening filter. Only for the purposes of this section, many can continue to complete the work, some other digital image processing methods further implemented, such as image smoothing, image matrix (K-L transform, Fourier transform, wavelet transform), image dilation and erosion, image geometric transformations (translation, rotation, scaling, etc.), and various image coding of the read and write and display. This part can be formation a Dedicated image processing platform.

About "face recognition", in the understanding of the various methods of face recognition, select the image histogram difference compared to achieve. The method can achieve better face classification, but higher demands on the face image, there is only the standard face database of Orlando in the image to be tested, so it can obtain higher recognition rate. And the face images collected in real life will be affected by many factors, the recognition rate it may not be able to meet the requirements of the. If we want to further improve the recognition rate and the scope of application, still need to be combined with other algorithms, such as PCA, neural network and so on.

\section{Reference}

[1] Wang Yunhong, Wei Fan, Tan Tieniu. A face recognition algorithm based on the global and local features of subspace face recognition [J]. Journal of electronics, 2015,28 (10):1657-1662.

[2] Zhang An, Zhuang Zhemin. Research on face recognition based on optimized LDA algorithm [J]. Chinese Journal of image and graphics, 2014,29 (9):2047-2049.

[3] bell. The realization of face recognition system based on Gabor wavelet [J]. Chinese Journal of image and graphics, 2015,23 (3): (in Chinese).

[4] Zhang Yankun, Liu Chongqing. A novel method of face recognition based on [J]. LDA Journal of automation, 2013,22 (5):327-330.

[5] Cao Lin, Wang Dongfeng, Liu Xiaojun, Zou Mouyan. 2D Gabor Wavelet Face Recognition Algorithm Based on [J]. Journal of electronics, 2010,28 (3) 490-494.

[6] Jiao Feng, Shan Shiguang, Guo Qin Cui, Gao Wen, Jin Tao Li. Based on local feature analysis of face recognition method [J]. Journal of automation, 2003,15 (1).

[7] Wangmeng Zuo, Kuanquan Wang, David Zhang, Hongzhi Zhang. Combination of two novel LDA-based methods for face recognition[C].Proceedings of the IEEE, 2007:735-742. 